\title{
Validity and reliability of a home-based, guardian- conducted video voiding test for voiding evaluation after hypospadias surgery
}

\author{
Jae Hyeon Han ${ }^{1}$ (i), Jang Hui Lee ${ }^{2}$ (i), Jaebeom Jun ${ }^{2}$ (i), Min Uk Park² ${ }^{2}$, Je Seong Lee ${ }^{3}$, Sungchan Park ${ }^{4}$, \\ Sang Hoon Song ${ }^{2}$ (id, Kun Suk Kim² ${ }^{2}$ \\ ${ }^{1}$ Department of Urology, Korea University Ansan Hospital, Korea University College of Medicine, Ansan, ${ }^{2}$ Department of Urology, Asan Medical Center, University of Ulsan \\ College of Medicine, Seoul, Korea, ${ }^{3}$ Eastern Virginia Medical School, Norfolk, VA, USA, ${ }^{4}$ Department of Urology, Ulsan University Hospital, University of Ulsan College of \\ Medicine, Ulsan, Korea
}

Purpose: To investigate the validity and reliability of a home-based, guardian-conducted video voiding test for assessing postoperative voiding function after hypospadias surgery.

Materials and Methods: In a single center, patients who had undergone urethroplasty by a single surgeon and postoperative uroflowmetry and video voiding tests conducted between 2008 and 2016 were retrospectively reviewed. Urinary stream was categorized into five grades by three pediatric urologists in a blinded manner. The primary outcome was statistical correlation across raters as measured by Spearman correlation coefficient to validate the interpretation of the video voiding test. The secondary outcome was the reliability of the voiding video test compared with maximum urinary flow rate assessed by uroflowmetry.

Results: Thirty-one patients with hypospadias were enrolled. The patients' average ages were $12.3 \pm 3.2$ months (range, 8-21 months) and 42.8 \pm 3.9 months (range, 35-48 months) at the time of surgery and voiding video tests, respectively. Hypospadias was anterior, penile, and proximal in 1 (3.2\%), 18 (58.1\%), and 12 (38.7\%) patients, respectively. The number of patients with each voiding stream grade was as follows: very poor, 4; poor, 4; fair, 13; good, 4; and very good, 6 . All intraclass correlation coefficients of the stream grade among the three observers were $>0.95$. Correlation between the maximum flow rate obtained by use of conventional uroflowmetry and the video voiding stream grade was validated (rho $0.778, \mathrm{p}<0.001$ ).

Conclusions: The home-based guardian-conducted video voiding test is easy to perform and the present results demonstrate its validity and reliability for assessing patients' post-urethroplasty voiding pattern.

Keywords: Hypospadias; Urethra; Urination; Video recording

This is an Open Access article distributed under the terms of the Creative Commons Attribution Non-Commercial License (http://creativecommons.org/licenses/by-nc/4.0) which permits unrestricted non-commercial use, distribution, and reproduction in any medium, provided the original work is properly cited.

\section{INTRODUCTION}

Urethral stricture is the second most common complication of hypospadias surgery [1,2]. In general, assessments of functional outcome are performed on the basis of patients' complaints about stream of micturition and the results of uroflowmetry tests after urethroplasty [3]. The measurement of urinary flow is a simple and noninvasive procedure that

Received: 28 November, 2019 • Accepted: 1 March, 2020

Corresponding Author: Kun Suk Kim (iD https://orcid.org/0000-0001-8982-6922

Department of Urology, Asan Medical Center, University of Ulsan College of Medicine, 88 Olympic-ro 43-gil, Songpa-gu, Seoul 05505, Korea

TEL: +82-2-3010-3736, FAX: +82-2-477-8928, E-mail: kskim2@amc.seoul.kr 
provides an objective evaluation of the urinary stream, and it has been used for a long time in urologic practice [4,5].

Even after patients acquire an appropriate caliber of neourethra after repair of hypospadias, the peak flow rate can still be reduced $[4,6]$. In the pediatric population, the maximal flow rate (Qmax) is significantly related to the voided volume, and nomograms of expected flow rates have been developed in pediatrics for this reason $[7,8]$. For pediatric patients, however, uroflowmetry is occasionally not easy to perform repeatedly when voiding volume is insufficient. Therefore, the results of a single test may not reflect the patients' usual urination. We thus considered the use of a video device to support our clinical interpretation of voiding patterns after hypospadias surgery in addition to the conventional uroflowmetry test.

A previous study in which parents video recorded their children's urination showed that this method decreases hospital anxiety in children and reduces clinic time at follow-up visits [9]. The authors showed that the prerecorded voiding video could be used to evaluate the voiding pattern instead of the uroflowmetry test results. However, no study has been conducted to determine whether home voiding video affects the surgeon's decision-making process at follow-up.

We hypothesized that the video voiding test would be of great support in diagnosing a patient's voiding pattern. We therefore evaluated the validity and reliability of a homebased, guardian-conducted video voiding test in investigating postoperative voiding function.

\section{MATERIALS AND METHODS}

\section{Study population}

This retrospective study protocol was approved by the Institutional Review Board of Asan Medical Center (approval number: 2019-1495) and complied with the principles of the Declaration of Helsinki. The requirement for informed consent was waived owing to the retrospective design. Between 2008 and 2016, consecutive patients with hypospadias who underwent urethroplasty in the pediatric urology department of a single tertiary center were screened retrospectively. Children were included in this study only if they were toilet trained, were able to void volitionally for a uroflowmetry test, and were required to undergo a voiding video taken at home by their guardians, who would send the video by e-mail to the physician. We excluded children who did not return for follow-up, or children with $<50 \mathrm{~mL}$ of voided volume during the uroflowmetry test. A total of 31 pediatric patients were enrolled in our study.

\section{Surgical procedures}

Hypospadias repair was performed by tubularized incised plate (TIP) urethroplasty for mid- to susceptible proximal penile hypospadias without severe curvature [10]. Staged urethroplasty was performed for proximal hypospadias with severe curvature [11,12]. All surgical procedures were ultimately determined in the operating room, although they were provisionally determined in the outpatient clinic. An erection test was performed to determine whether chordee was present; thereafter, the penile curvature was corrected by dorsal albuginea plication. For staged urethroplasty, the second operation, tubularization of neourethra, was performed 6 months after the primary surgery.

\section{Patients' postoperative follow-up with uroflowmetry and voiding video}

We followed patients after toilet training. At the first uroflowmetry test, parents were requested to obtain a voiding video. Parents used a cellular phone to take the voiding videos while their children were urinating. All videos were captured under calm circumstances with the boys in the standing position during the entire micturition. Parents were asked to take the best three videos showing enough voided volume and a good stream before coming to the outpatient clinic. Patients who had a good voiding pattern (bell or plateau shaped) on uroflowmetry with a voiding video grade of more than fair were recommended to return to the clinic 6 to 12 months later. Patients with a low Qmax with an obstructive pattern were recommended to visit the clinic within $<3$ months. Patients were requested to undergo uroflowmetry at each outpatient office visit. For all patients with an unsatisfactory voiding result, the parents were asked to carefully observe their children's voiding.

\section{Video voiding test and interpretation}

Three observers performed the evaluation retrospectively without knowing the patient's clinical course. Voiding flow was categorized into five grades (very poor, poor, fair, good, and very good) by three urologists trained in pediatric urology (a: fellowship doctor, b: resident doctor, and c: pediatric urology professor) who viewed the videos on a desktop computer monitor. Before the urologists evaluated the videos, we presented a standardized voiding video of patient 1 and patient 2 (Fig. 1), each representing very poor and very good grade, respectively. The observers reviewed the video once for the entire micturition. A uroflowmetry test was performed to assess the voiding function of the patients in the outpatient clinic within a $<2$-week interval from the video voiding test. Uroflowmetry data were measured on a rotat- 


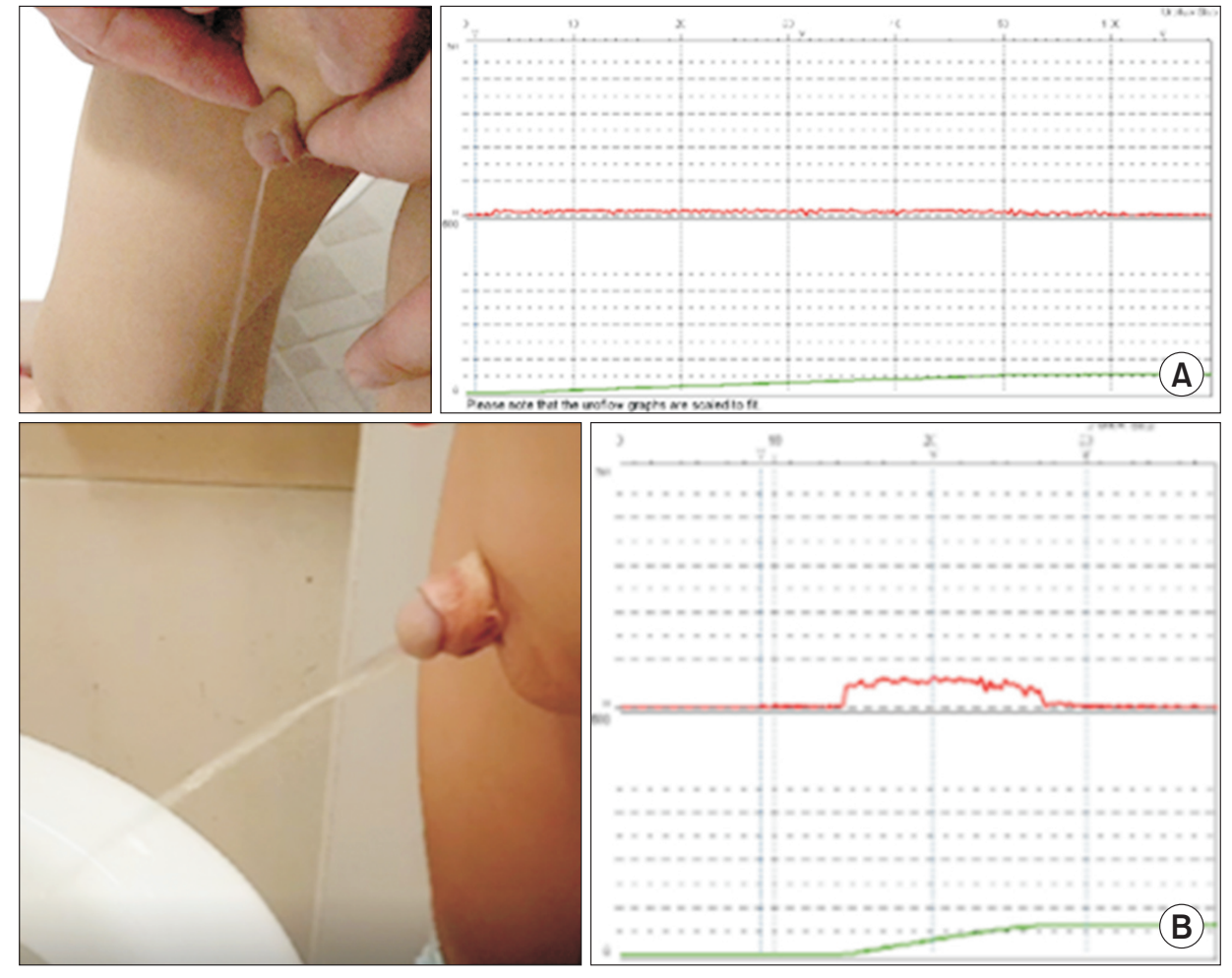

Fig. 1. (A) Very poor stream grade; voided volume $(\mathrm{mL}) / \mathrm{Qmax}(\mathrm{mL} / \mathrm{s}) /$ residual urine volume $(\mathrm{mL})$ was $68 / 2 / 31$. (B) Very good stream grade; voided volume $(\mathrm{mL}) / \mathrm{Qmax}$ $(\mathrm{mL} / \mathrm{s}) /$ residual urine volume $(\mathrm{mL})$ was $76 / 6.6 / 0$. Parents and patients consented for these photographs to be used in medical publications. Qmax, maximal flow rate. ing disk system, and each patient's urinary flow pattern was determined; in addition, the Qmax and average flow rate (Qave) were measured. Qmax was calculated from the flow curves in $\mathrm{mL} / \mathrm{s}$. The urinary flow patterns were classified as bell-shaped, plateau, or intermittent. The validity and reliability of the video voiding test were evaluated on the basis of the measured grade from the three observers and the Qmax (mL/s) of uroflowmetry.

\section{Follow-up strategy of patients with obstructive voiding pattern}

During the follow-up, patients with a low Qmax $(<3 \mathrm{~mL} /$ s) and obstructive flow pattern were recommended to undergo surgical repair. All urethrectomies were performed by urethroscopic surgery with the patient under general anesthesia. With a pediatric urethroscope, a cold knife was used to make an incision in the 12-o'clock direction of the urethra. Afterward, the uroflowmetry test was performed again to assess for improvement of voiding.

\section{Statistical analysis}

Continuous variables are expressed as means \pm standard deviations. Categorical variables are shown as percentages. All p-values $<0.05$ were considered statistically significant. The Kruskal-Wallis test was used to assess the validity of the voiding video stream grade. The intraclass correlation coefficient was calculated with a kappa value. Data were analyzed by using IBM SPSS Statistics ver. 19.0 (IBM Corp., Armonk, NY, USA).

\section{RESULTS}

The patients' baseline characteristics are shown in Table 1. The patients' average ages were $12.3 \pm 3.2$ months (range, 8-21 months) and 42.8 \pm 3.9 months (range, 35-48 months) at the time of surgery and the voiding video tests, respectively. The mean follow-up period was $53.4 \pm 26.5$ months (range, 21-127 months). Hypospadias type was anterior, penile, and proximal in 1 (3.2\%), 18 (58.1\%), and 12 (38.7\%) patients, respectively. Uroflowmetry data were successfully obtained from 31 patients, all of whom had no early postoperative complications and urinated $>50 \mathrm{~mL}$. All patients underwent a postoperative uroflowmetry test more than once.

Fig. 1 shows examples of two patients' video voiding tests that were evaluated as very poor and very good, respectively. Patient 1 underwent TIP urethroplasty at 11 months of age for penoscrotal hypospadias without severe curvature (Fig. 1A). His uroflowmetry data showed a flat pattern suggesting obstruction with a low maximal flow $(2 \mathrm{~mL} / \mathrm{s}$, voided volume of $68 \mathrm{~mL}$, and residual volume of $31 \mathrm{~mL}$ ) at 39 months of age. He showed a straining pattern of voiding. All observers gave him a grade of very poor. After more than two uroflowmetry tests had been performed, he underwent urethroscopic internal urethrotomy for relief of urethral stricture. 
Table 1. Patients' baseline characteristics

\begin{tabular}{|c|c|c|}
\hline Variable & Value & Type of surgery and surgical site \\
\hline No. of patients & 31 & \\
\hline Age at urethroplasty & $12.3 \pm 3.2(8-21)$ & \\
\hline \multicolumn{3}{|l|}{ Type of hypospadias } \\
\hline \multicolumn{3}{|l|}{ Anterior } \\
\hline Glandular & $1(3.2)$ & MAGPI 1/1 \\
\hline \multicolumn{3}{|l|}{ Penile } \\
\hline Distal penile & $9(29.0)$ & TIP 9/9 \\
\hline Mid-penile & $5(16.1)$ & TIP 4/5, duplay $1 / 5$ \\
\hline Proximal penile & $4(12.9)$ & TIP 3/4, onlay island flap $1 / 4$ \\
\hline \multicolumn{3}{|l|}{ Proximal } \\
\hline Penoscrotal & $11(35.5)$ & TIP 11/11 \\
\hline Scrotal & $1(3.2)$ & Staged urethroplasty $1 / 1$ \\
\hline Age at uroflowmetry and video voiding tests (mo) & $42.8 \pm 3.9(35-48)$ & \\
\hline $\mathrm{VV}(\mathrm{mL})$ & $110.6 \pm 53.6(51.8-262.7)$ & \\
\hline PVR (mL) & $17.6 \pm 18.7(0-72.0)$ & \\
\hline Qmax (mL/s) & $6.8 \pm 4.7(1.2-24.4)$ & \\
\hline PVR/VV (\%) & $15.4 \pm 16.1(0-54.4)$ & \\
\hline No. of internal ureteroscopic urethrotomies & $5(16.1)$ & $\begin{array}{l}\text { Distal penile } 2 / 5 \\
\text { Mid penile } 1 / 5 \\
\text { Penoscrotal } 1 / 5 \\
\text { Scrotal } 1 / 5\end{array}$ \\
\hline Follow-up duration (mo) & $53.4 \pm 26.5(21-127)$ & \\
\hline
\end{tabular}

Values are presented as number only, mean \pm standard deviation (range), or number (\%).

MAGPI, meatal advancement and glanduloplasty; TIP, tubularized incised plate; VV, voiding volume; PVR, postvoiding residual; Qmax, maximal flow rate.

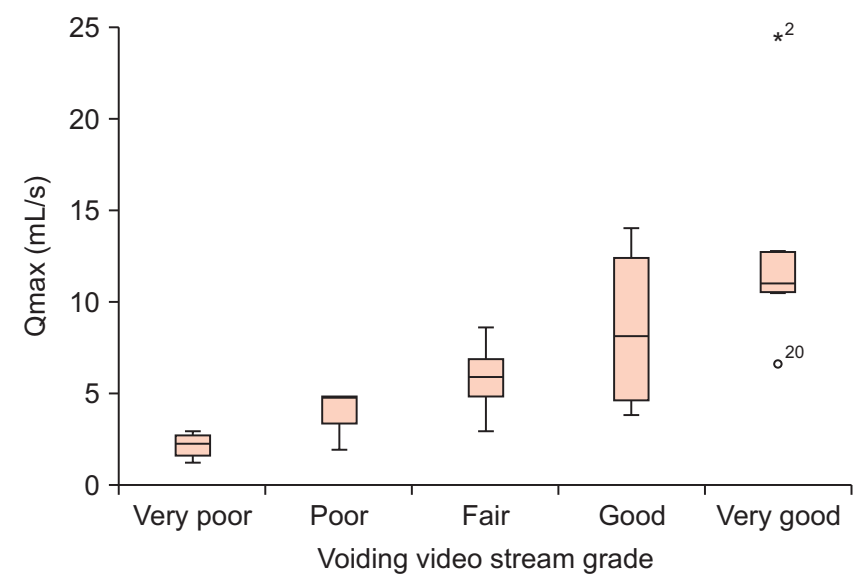

Fig. 2. Mean maximal flow rate $(\mathrm{Qmax})(\mathrm{mL} / \mathrm{s})$ by grade of the voiding video stream (Spearman $r h o=0.778, p<0.001)$.

Patient 2 underwent TIP urethroplasty for distal penile hypospadias at 13 months of age. The result of his uroflowmetry test after toilet training showed a plateau pattern of voiding with a voided volume of $76.6 \mathrm{~mL}$ and a Qmax of 6.6 $\mathrm{mL} / \mathrm{s}$ with no residual urine at 33 months of his age (Fig. 1B). His stream was graded as very good by all reviewers.

The validity of the voiding stream grade among the three observers is demonstrated in Fig. 2. Because of some
Table 2. Intraclass correlation coefficients of the stream grade of the video voiding test among the three observers

\begin{tabular}{lcccc}
\hline $\begin{array}{c}\text { Video voiding test } \\
\text { session }\end{array}$ & ICC & $95 \% \mathrm{Cl}$ & F test & p-value \\
\hline 1st video evaluation & 0.971 & $0.948-0.985$ & 34.839 & $<0.001$ \\
2nd video evaluation & 0.965 & $0.937-0.982$ & 28.857 & $<0.001$ \\
3rd video evaluation & 0.967 & $0.940-0.983$ & 30.503 & $<0.001$ \\
\hline
\end{tabular}

Reader 1: fellowship doctor $(\mathrm{JHH})$, reader 2: residency doctor $(\mathrm{JHL})$, reader 3: professor (KSK).

ICC, intraclass correlation coefficient; $\mathrm{Cl}$, confidence interval.

disagreement in grading among the three judges, we chose a representative grade for study analysis. The number of patients for each grade were as follows: very poor, 4; poor, 4; fair, 13; good, 4; and very good, 6. The mean $\mathrm{Qmax}(\mathrm{mL} / \mathrm{s})$ of the representative voiding stream grades were as follows: 2.15 for very poor, 4.05 for poor, 5.83 for fair, 8.5 for good, and 127 for very good. The Kruskal-Wallis H test showed a statistically significant difference between the Qmax of the representative voiding stream grades $\left(\chi^{2}(2)=18.584, p=0.001\right)$.

Table 2 shows the reliability of the three observers for the three videos (first, second, and third) from each patient. For all videos, intraclass correlation coefficients of the stream grade of the video voiding test among the three 
observers were high $(0.971,0.965$, and 0.967$)$. A correlation between the maximum flow rate obtained by the conventional uroflowmetry method and video stream grade was validated by the Spearman bivariate analysis with a rho of 0.778 $(p<0.001)$.

\section{DISCUSSION}

In the era of easy video recording with cellular phones, interest in evaluating and examining patients by use of this technology is high [9]. This study investigated the clinical value of performing a video voiding test after hypospadias surgery, as it is helpful for surgeons to observe patients' urination and detect urethral stricture by a method other than only the uroflowmetry report. Although surgeons can observe the voiding pattern during patients' urination in a uroflowmetry test, it is often difficult to observe patients' voiding directly owing to many factors, including a busy clinical practice, insufficient voided volume with a low Qmax, and patient anxiety. Video recording of micturition in normal conditions is not biased by artificial conditions (as occurs when the patient is asked to void in a stressful medicalized situation), which may give efficacious information for detecting obstructive voiding patterns after hypospadias.

For diagnosing lower urinary tract dysfunction in male patients, one article previously introduced a novel video voiding device to document key voiding parameters like instantaneous flow rate [13]. However, use of that device is not practical at home. In our practice, surgeons usually ask parents at the time of the outpatient clinic visit to take video after the child is toilet trained. Our study showed the reliability and validity of a video voiding test for evaluating patients' voiding stream pattern after hypospadias surgery. We analyzed uroflowmetry to evaluate the role of Qmax in providing valuable information for following up patients. Even though other parameters including residual urine volume were studied, no significant correlation could be established.

The urethra, if reconstructed, should have an adequate caliber and be enlarged as the patient ages [7]. However, urethral obstruction due to stricture is a common complication of hypospadias surgery, and the urethral meatus or anastomotic site of the neourethra is a possible site of obstruction. In cohort of TIP urethroplasty, a meta-analysis of hypospadias complications showed meatal stenosis complication rates of $3.6 \%, 4.4 \%$, and $8.3 \%$ for primary distal, primary proximal, and secondary hypospadias, respectively, after more than 1 year of follow-up [14]. The incidence of urethral strictures was $13 \%, 2.0 \%$, and $3.0 \%$ for primary distal, primary proxi- mal, and secondary hypospadias, respectively [14]. Often, no apparent symptoms are present until complete obstruction occurs [15].

Urinary flow rates after hypospadias surgery were first reported in older patients in the 1970s [4]. The weak flow rate is caused by stenosis, low intravesical pressure, rigidity, and lack of elasticity of the neourethra, pseudo-obstruction, and lack of a corpus spongiosum [4]. In our study, patients with hypospadias also showed a decreased urine flow rate, and they were characterized by a fractionated flow pattern. Uroflowmetry data include several parameters as defined by the International Children's Continence Society [16]. Authors have reported that patients with hypospadias show abnormal (though subclinical) flow patterns postoperatively $[4,17,18]$. Some authors have also noted a frail correlation between flow and clinical symptoms [3]. Because of this, a single uroflowmetry test or single voiding video test cannot represent the long-term result of hypospadias surgery. Children with an obstructed or borderline voiding pattern must be followed until young adulthood.

In uroflowmetry, Qmax is affected not by voided volume, but rather by total bladder volume including residual urine volume. Uroflow is based mainly on the weight of the stream falling on the rotating drum, which is objectively translated into speed in $\mathrm{mL} / \mathrm{s}$. This is completely absent in home-based video voiding. So, what looks like a nice flow in a video could be very misleading if we do not measure the amount voided. Whether due to a small amount of voided volume without obstruction or whether due to real obstruction with large residual urine, it is difficult to obtain an accurate assessment by predicting the flow rate with only subjective visual inspection without knowing residual urine volume. Even though we excluded children with $<50 \mathrm{~mL}$ of voided volume during the uroflowmetry test as a comparative marker, the lack of measurement of volume of videorecorded voiding will lead to falsely including patients with low volumes of $<50 \mathrm{~mL}$. For this reason, we asked the guardians to obtain the three best videos to reduce errors.

Among all patients, five patients (16.1\%) had urethral stricture with obstructive symptoms within the mean 50-month follow-up. They underwent direct-vision internal urethrotomy afterwards [19]. Three of them were graded as poor or less and two of them were graded as fair on the initial video voiding test. At the initial evaluation of voiding, patients who had a good or very good grade did not require a second intervention. The postoperative follow-up schedule was recommended initially at 6 months and then after toilet training until puberty [20]. In our series, to recognize patients' obstructive symptom presentation and obtain infor- 
mative uroflowmetry, it was essential to observe a voiding pattern after toilet training. We categorized voiding videos according to the initial presenting position of hypospadias, single or staged urethroplasty, and presence of curvature. However, there was no correlation of severity of hypospadias with voiding pattern.

There were some limitations to our study. In this article, we attempted to prove the validity and reliability of the home-based video to evaluate the uroflow after hypospadias repair. This was a retrospective study with a low number of 31 patients. For a more reliable outcome, a larger cohort and prospective study will be needed. However, our results showed the reliability and usefulness of the video voiding test with significant statistics. Second, each of the three reviewers subjectively measured and in conclusion confirmed the reliability of the video voiding test grade. We proposed two video clips as a milestone for the grading system. For this reason, further discussion on selection of the standard video clip is essential to improve our test. With the guidelines we presented here, such discussion to establish a standardized concept would be easy to accomplish.

The current study did not investigate follow-up serial video voiding tests after the initial results. Among the patients who underwent urethral stricture repair afterward, we found that the primary evaluation of voiding does not always indicate the long-term follow-up result. There are a few studies of the long-term evaluation of the TIP urethroplasty/Snodgrass technique [21,22]. According to those results, most of the urodynamic problems with the TIP urethroplasty technique may revert to normal spontaneously in the late postoperative period. In our study, before performing an urethrotomy, all patients were symptomatic and were followed for a sufficient length of time. Even with the limitations noted above, to our knowledge, our study is the first to show the validity and reliability of a video voiding test for the evaluation of voiding patterns after hypospadias repair.

\section{CONCLUSIONS}

The home-based, guardian-conducted video voiding test is a validated and reliable method for evaluating children's voiding stream with ease and repeatability at home. This test will serve as a supportive tool to conventional uroflowmetry and can help physicians assess children's post-urethroplasty urination. Our findings are helpful in showing that video recording of micturition in normal conditions is not biased by "artificial" conditions and can be efficacious in detecting obstructive voiding patterns after hypospadias.

\section{CONFLICTS OF INTEREST}

The authors have nothing to disclose.

\section{AUTHORS' CONTRIBUTIONS}

Research conception and design: Kun Suk Kim and Jae Hyeon Han. Data acquisition: Jae Hyeon Han, Jang Hui Lee, Jaebeom Jun, Min Uk Park, Je Seong Lee, Sang Hoon Song, and Kun Suk Kim. Statistical analysis: Jae Hyeon Han, Jang Hui Lee, and Kun Suk Kim. Data analysis and interpretation: Jae Hyeon Han, Sungchan Park, Sang Hoon Song, and Kun Suk Kim. Drafting of the manuscript: Jae Hyeon Han, Je Seong Lee, and Kun Suk Kim. Critical revision of the manuscript: Jae Hyeon Han, Sungchan Park, Sang Hoon Song, and Kun Suk Kim. Obtaining funding: none. Administrative, technical, or material support: Jae Hyeon Han, Jang Hui Lee, Jaebeom Jun, Min Uk Park, and Je Seong Lee. Supervision: Kun Suk Kim. Approval of the final manuscript: all authors.

\section{REFERENCES}

1. de Badiola F, Anderson K, Gonzalez R. Hypospadias repair in an outpatient setting without proximal urinary diversion: experience with 113 urethroplasties. J Pediatr Surg 1991;26:4614; discussion 464-5.

2. Hsiao KC, Baez-Trinidad L, Lendvay T, Smith EA, Broecker $\mathrm{B}$, Scherz H, et al. Direct vision internal urethrotomy for the treatment of pediatric urethral strictures: analysis of $50 \mathrm{pa}-$ tients. J Urol 2003;170:952-5.

3. Spinoit AF, Hoebeke P. Paediatrics: urinary flow rate after TIP urethroplasty for hypospadias. Nat Rev Urol 2015;12:477-8.

4. Page RE, Akin Y. Assessment of urine flow in hypospadias. Br J Plast Surg 1978;31:313-6.

5. Hjalmas K, Hoebeke PB, de Paepe H. Lower urinary tract dysfunction and urodynamics in children. Eur Urol 2000;38:65565.

6. Idzenga T, Kok DJ, Pel JJ, van Mastrigt R, Wolffenbuttel KP. Is the impaired flow after hypospadias correction due to increased urethral stiffness? J Pediatr Urol 2006;2:299-303.

7. Perera M, Jones B, O'Brien M, Hutson JM. Long-term urethral function measured by uroflowmetry after hypospadias surgery: comparison with an age matched control. J Urol 2012;188(4 Suppl):1457-62.

8. Pernkopf D, Plas E, Lang T, Daha K, Kubin K, Treu T, et al. Uroflow nomogram for male adolescents. J Urol 2005;174(4 Pt 1):1436-9; discussion 1439.

9. Türk E, Güven A, Karaca F, Edirne Y, Karaca I. Using the 
parents' video camera for the follow-up of children who have undergone hypospadias surgery decreases hospital anxiety of children. J Pediatr Surg 2013;48:2332-5.

10. Chen SC, Yang SS, Hsieh CH, Chen YT. Tubularized incised plate urethroplasty for proximal hypospadias. BJU Int 2000;86: 1050-3.

11. Zheng DC, Yao HJ, Cai ZK, Da J, Chen Q, Chen YB, et al. Twostage urethroplasty is a better choice for proximal hypospadias with severe chordee after urethral plate transection: a singlecenter experience. Asian J Androl 2015;17:94-7.

12. Snodgrass W, Bush N. Staged tubularized autograft repair for primary proximal hypospadias with 30-degree or greater ventral curvature. J Urol 2017;198:680-6.

13. Shokoueinejad M, Alkashgari R, Mosli HA, Alothmany N, Levin JM, Webster JG. Video Voiding Device for diagnosing Lower Urinary Tract Dysfunction in men. J Med Biol Eng 2017; 37:474-83.

14. Pfistermuller KL, McArdle AJ, Cuckow PM. Meta-analysis of complication rates of the tubularized incised plate (TIP) repair. J Pediatr Urol 2015;11:54-9.

15. Scherz HC, Kaplan GW, Packer MG, Brock WA. Post-hypospadias repair urethral strictures: a review of 30 cases. J Urol 1988;140(5 Pt 2):1253-5.
16. Van Batavia JP, Combs AJ. The role of non-invasive testing in evaluation and diagnosis of pediatric lower urinary tract dysfunction. Curr Urol Rep 2018;19:34.

17. Piplani R, Aggarwal SK, Ratan SK. Role of uroflowmetry before and after hypospadias repair. Urol Ann 2018;10:52-8.

18. Kaya C, Kucuk E, Ilktac A, Ozturk M, Karaman MI. Value of urinary flow patterns in the follow-up of children who underwent Snodgrass operation. Urol Int 2007;78:245-8.

19. Wang X, Tang Y, Mao Y, Qin D, Chen S. [Reoperation methods and effectiveness of urethral stricture after urethroplasty of hypospadias]. Zhongguo Xiu Fu Chong Jian Wai Ke Za Zhi 2019;33:223-6. Chinese.

20. Snodgrass W, Villanueva C, Bush NC. Duration of follow-up to diagnose hypospadias urethroplasty complications. J Pediatr Urol 2014;10:208-11.

21. Erickson BA, Breyer BN, McAninch JW. The use of uroflowmetry to diagnose recurrent stricture after urethral reconstructive surgery. J Urol 2010;184:1386-90.

22. Hueber PA, Antczak C, Abdo A, Franc-Guimond J, Barrieras D, Houle AM. Long-term functional outcomes of distal hypospadias repair: a single center retrospective comparative study of TIPs, Mathieu and MAGPI. J Pediatr Urol 2015;11:68.e1-7. 